\title{
Imaging technology in mice enhances human brain research
}

\author{
Mohammad R. N. Avanaki, Jun Xia, Hanlin Wan, \\ Adam Q. Bauer, Joseph P. Culver, and Lihong V. Wang
}

Photoacoustic tomography offering a large field of view and high spatial resolution enables, for the first time, noninvasive imaging of restingstate functional connectivity in the murine brain.

One of many approaches to studying neurological function involves imaging the brain's functional connectivity, an observational analysis of neuronal activity in different parts of the brain. Recent research suggests that fluctuations in the activity of separate groups of neurons are interactively correlated, ${ }_{1}^{1}$ although they do not affect one another's activity directly. Resting-state functional connectivity (RSFC) imaging is one technique that is emerging to identify low-frequency, spontaneous cerebral fluctuations in hemodynamics-such as blood oxygenation and total hemoglobin concentration-that are associated with these functional connections. ${ }^{2}$

A hallmark of functional organization in the brain's cortex is the striking bilateral symmetry of corresponding functional regions in the left and right hemispheres. This symmetry also exists in spontaneous resting-state hemodynamics, where strong correlations are found interhemispherically-between hemispheres-within bilaterally homologous regions, as well as intrahemispherically-inside each hemisphere-within the same functional regions. ${ }^{3}$

Murine (mouse) models are increasingly being used for human brain disease studies, possibly owing to the similarity in the structures of the murine and human brains. This has created a need for a new functional imaging modality because current imaging techniques cannot be easily applied to mice. For instance, in functional connectivity magnetic resonance imaging (fcMRI), resting-state brain activity is determined via blood-oxygen-level-dependent (BOLD) signal contrast, which originates mainly from deoxyhemoglobin. ${ }^{4}$ The correlation analysis central to functional connectivity requires a high signalto-noise ratio (SNR). However, achieving a sufficient SNR is challenging owing to the need both for high magnetic fields and small voxel size for imaging the mouse brain and for the

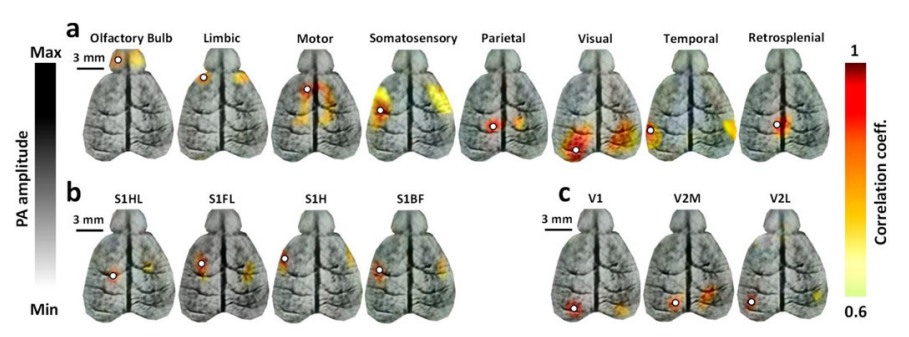

Figure 1. Functional connectivity maps in a murine brain acquired noninvasively by functional connectivity photoacoustic tomography $\left(f_{c} P A T\right)$. Correlation maps of (a) the eight main functional regions, (b) the four subregions of the somatosensory cortex, and (c) the three subregions of the visual cortex. PA: Photoacoustic. White circles: Seed regions. S1HL: Primary somatosensory cortex, hindlimb region. S1FL: Primary somatosensory, forelimb region. S1H: Primary somatosensory, head region. S1BF: Primary somatosensory, barrel field. V1: Primary visual cortex. V2M: Secondary visual cortex, medial region. V2L: Secondary visual cortex, lateral region.

complexity of compensating for field inhomogeneities caused by tissue-bone or tissue-air boundaries.

Researchers have recently introduced functional connectivity mapping with optical intrinsic signal imaging (fcOIS) as an alternative approach in mice. ${ }^{5}$ However, due to the diffusion of light in tissue, the spatial resolution of fcOIS is limited, and experiments thus far performed used an exposed skull preparation, which increases the complexity of longitudinal imaging.

Using optical excitation and acoustic detection, we developed a functional connectivity photoacoustic tomography (fcPAT) system that, for the first time, allows noninvasive imaging of RSFC — with a large field of view and high spatial resolutionin the murine brain. ${ }^{6}$ We observed bilateral correlations in eight functional regions, including the olfactory bulb and the limbic, parietal, somatosensory, retrosplenial, visual, motor, and temporal regions, as well as in several subregions (see Figure 1). ${ }^{6}$ The borders and locations of these regions agreed well with the Paxinos murine brain atlas.? 

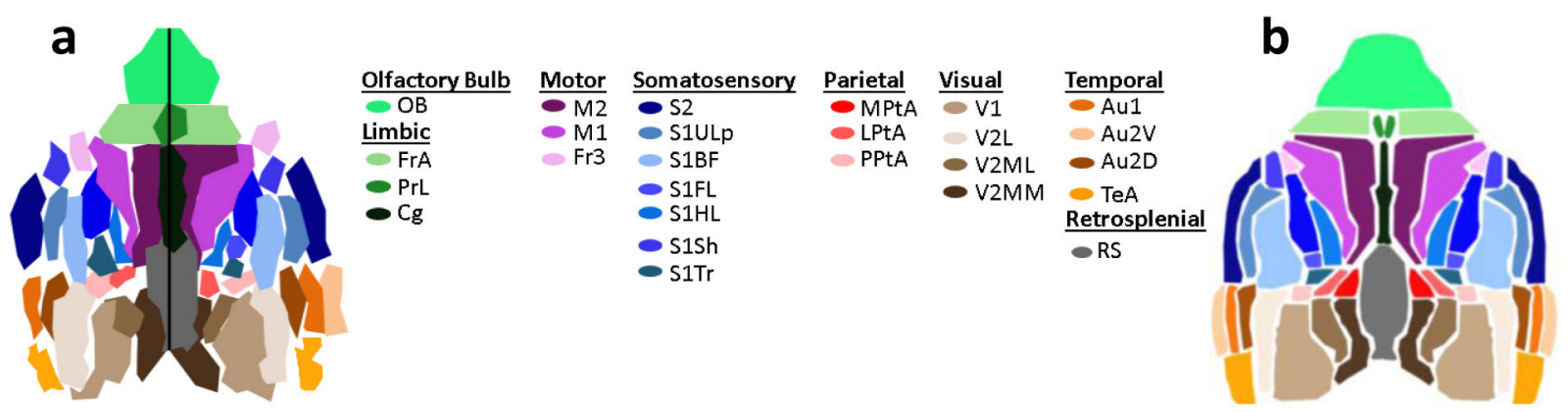

Figure 2. (a) Parcellation map of the murine brain described in Figure 1. (b) Corresponding functional regions from the Paxinos histological atlas. ${ }^{7}$ Au1: Primary auditory cortex. Au2D: Secondary auditory, dorsal area. Au2V: Secondary auditory, ventral area. Cg: Cingulate. Fr3: Frontal cortex area 3. FrA: Frontal association. LPtA: Lateral parietal association. M1: Primary motor cortex. M2: Secondary motor cortex. MPtA: Medial parietal association. OB: Olfactory bulb. PPtA: Posterior parietal association. PrL: Prelimbic. RS: Retrosplenial area. S1ULp: Primary somatosensory, upper lip region. S1BF: Primary somatosensory, barrel field. S1FL: Primary somatosensory, forelimb region. S1HL: Primary somatosensory cortex, hindlimb region. S1Sh: Primary somatosensory, shoulder region. S1Tr: Primary somatosensory cortex, trunk region. S2: Secondary somatosensory. TeA: Temporal association cortex. V1: Primary visual cortex. V2MM: Secondary visual cortex, mediomedial region. V2ML: Secondary visual cortex, mediolateral region. V2L: Secondary visual cortex, lateral region.

We developed a parcellation algorithm to recreate the functional divisions within the murine brain in a data-driven manner (see Figure 2). In the parcellated image, most of the regions and subregions were evident. Regions such as the olfactory bulbs, motor and visual cortices, and retrosplenial and temporal regions were more pronounced in the parcellated image, while parcels were absent for some of the parietal and somatosensory subregions. ${ }^{6}$ By subjecting the mouse to alternating hyperoxic and hypoxic conditions, we observed strong and weak functional connectivities, respectively. In addition, we simultaneously acquired vascular images. ${ }^{6}$

Our studies show fcPAT to be a promising noninvasive technique for functional connectivity imaging in the murine brain. Translation of fcPAT to large animals and humans is also possible, as photoacoustic techniques have been used successfully to image through monkey and human skulls. The lower cost of fCPAT compared to fMRI can help support research efforts in many laboratories that formerly were unable to use functional neuroimaging in their ongoing studies of human disease. We are currently investigating the effect of Alzheimer's disease and also aging on the functional connectivity of the different regions and subregions.

\section{Author Information}

Mohammad R. N. Avanaki, Jun Xia, Hanlin Wan, Adam Q. Bauer, Joseph P. Culver and Lihong V. Wang Washington University

St. Louis, MO
Mohammad R. N. Avanaki received his PhD with Outstanding Achievement in medical image computing from the University of Kent. He is currently a postdoctoral fellow in Lihong V. Wang's laboratory. His research focuses on using high-resolution medical imaging modalities, including optical coherence tomography and photoacoustic technology in biomedical applications, and biomedical image computing.

Jun Xia earned his PhD at the University of Toronto and is currently a postdoctoral fellow at Washington University under the mentorship of Lihong V. Wang. His research interests focus on the development of novel biomedical imaging techniques, including photoacoustic imaging and ultrasonic imaging. He has published more than 20 peer-reviewed journal articles in photoacoustic and photothermal research.

Hanlin Wan received his BS in 2011 from the Johns Hopkins University and is currently pursuing a $\mathrm{PhD}$ at Washington University. His research focuses on the tracking and management of tumor motion in radiotherapy.

Adam Q. Bauer is a postdoctoral scholar at Washington University in the laboratory of Joseph P. Culver.

Joseph P. Culver is an associate professor in the Radiology Department of Washington University and director of the Optical Radiology Laboratory. 
Lihong V. Wang holds a Beare distinguished professorship. He has published 365 journal articles with an h-index of 84 (>28,000 citations) and delivered 380 keynote-plenary-invited talks. He serves as the editor-in-chief of the Journal of Biomedical Optics. He was awarded the Optical Society's Mees Medal, the National Institutes of Health Director's Pioneer Award, the Goodman Book Award, and the IEEE's Biomedical Engineering Award.

\section{References}

1. Brian R. White, Adam Q. Bauer, Abraham Z. Snyder, Bradley L. Schlaggar, Jin-Moo Lee, and Joseph P. Culver, Imaging of functional connectivity in the mouse brain, PloS ONE 6 (1), p. e16322, 2011.

2. Bharat Biswal, F. Zerrin Yetkin, Victor M. Haughton, and James S. Hyde, Functional connectivity in the motor cortex of resting human brain using echo-planar MRI, Magn. Reson. Med. 34 (4), pp. 537-541, 1995.

3. Michael D. Fox and Marcus E. Raichle, Spontaneous fluctuations in brain activity observed with functional magnetic resonance imaging, Nat. Rev. Neurosci. 8 (9), pp. $700-$ 711, 2007.

4. Jens Steinbrink, Arno Villringer, Florian Kempf, Daniel Haux, Stefanie Boden, and Hellmuth Obrig, Illuminating the BOLD signal: combined fMRI-fNIRS studies, Magn. Reson. Imag. 24 (4), pp. 495-505, 2006.

5. Adam W. Bero, Adam Q. Bauer, Floy R. Stewart, Brian R. White, John R. Cirrito, Marcus E. Raichle, Joseph P. Culver, and David M. Holtzman, Bidirectional relationship between functional connectivity and amyloid- $\beta$ deposition in mouse brain, J. Neurosci. 32 (13), pp. 4334-4340, 2012.

6. Mohammadreza Nasiriavanaki, Jun Xia, Hanlin Wan, Adam Q. Bauer, Joseph P. Culver, and Lihong V. Wang, High-resolution photoacoustic tomography of restingstate functional connectivity in the mouse brain, Proc. Nat'l Acad. Sci. USA 111 (1), pp. 21-26, 2014.

7. George Paxinos and Keith B. J. Franklin, The Mouse Brain in Stereotaxic Coordinates, 2nd ed., Academic Press, San Diego, 2003. 